\title{
Crisis Prevention and Control Management of the COVID-19 Pandemic
}

\author{
Yaxin Hou, Lian Zhang, Mengmeng Guo, Huifei Wang* \\ Graduate School, China People's Police University, Langfang, China \\ Email address: \\ felix75@126.com (Huifei Wang) \\ ${ }^{*}$ Corresponding author \\ To cite this article: \\ Yaxin Hou, Lian Zhang, Mengmeng Guo, Huifei Wang. Crisis Prevention and Control Management of the COVID-19 Pandemic. World \\ Journal of Public Health. Vol. 6, No. 3, 2021, pp. 121-127. doi: 10.11648/j.wjph.20210603.16
}

Received: July 11, 2021; Accepted: August 3, 2021; Published: August 23, 2021

\begin{abstract}
It is crucial that prevention and control of the COVID-19 global pandemic is summarized ahead of the next pandemic presumably, under the current severe pandemic situation. Foreign experience in COVID-19 crisis prevention and control management is introduced, and the main prevention and control strategies include isolation, mitigation, and inhibition. In addition, the experience gained from China's prevention and control of COVID-19 is summarized in terms of the whole national system, efficient joint prevention and control mechanism, cadre accountability system, simultaneous medical and non-medical measures, and timely, transparent and open Information. The enlightenment of epidemic crisis prevention and control management in the future is also discussed. (1) It is of great importance to mobilize the whole people to improve public health literacy and establish awareness of prevention and control. (2) Establishing public health crisis prevention and control platform and improving monitoring and early warning mechanism of public health crisis are emphasized, timely detection of the epidemic and doing a good job in early response. (3) Carrying out detection and monitoring, and implementing prevention and control measures are essential to establish a strict disease prevention and control system, such as overseas importation, safety protection, daily prevention and control of high-risk places. (4) It is essential to establish a strong command and support system, joint multi-department cooperation, strengthen international cooperation, formulate emergency plans, and do well in normalized and accurate prevention and control. The research described in the paper aims to provide reference for the prevention and control management of the COVID-19 global pandemic crisis.
\end{abstract}

Keywords: COVID-19 Pandemic, Prevention and Control, Crisis Management

\section{Introduction}

As a rare event, a pandemic is highly spread, with high incidence rate and propensity to relapse. It causes a large number of infections and deaths, which poses a great threat to social security, economic stability and human health [1]. There were three influenza pandemics in the 20th century, including H1N1 influenza pandemic in 1918-1919 [2], H2N2 influenza pandemic in 1957-1958, and H3N2 influenza pandemic in 1968-1969 [3]. Since the 21st century, Influenza SARS (2003), H1N1 (2009), MERS (2012), H7N9 (2009, 2013), Ebola virus (2014) pandemics occurred [4], and the COVID-19 pandemic in 2020. COVID-19 is a kind of infectious disease pandemic that caused by the novel coronavirus. In December 2019, some hospitals in Wuhan, Hubei Province reported the first case of COVID-19 to WHO after discovering pneumonia cases of unknown cause. In January 2020, Thailand, South Korea, Japan, the United States and other countries successively announced confirmed cases. Faced with COVID-19 that suddenly swept across the world, at the first time, no countries took effective measures to prevent and control the disease, and the epidemic spread rapidly. It is the Public Health Emergency of International Concern (PHEIC), and it also is the most serious public health event that Chinese people has suffered from since the founding of New China. It has had a great impact on global politics, economy, society, culture, production and other aspects, resulting in the global public health security crisis. The COVID-19 pandemic crisis management is not only China's problem but also the world's problem.

Based on the novel coronavirus pneumonia's serious harm, 
countries around the world, from the perspective of political and economic considerations, social governance models, social cultural differences, and in line with their actual conditions, adopted different COVID-19 prevention and control systems and institutions. South Korea took the Korean Disease Management Agency as the command center of the infectious disease crisis response system. It launched the response mechanism with the Central Epidemic Prevention Countermeasures Headquarters as the command center, the operation mechanism of the Central Disaster Safety Countermeasures Headquarters, and the mechanism of the Local Regional Disaster Safety Countermeasures Headquarters [5-6]. Singapore's health system had established strict infection control procedures and implemented in accordance with the rules. An interdepartmental working group led by the Ministry of Health, has been established to take charge of epidemic prevention and control, including the Ministry of National Development, the Ministry of Education, the Ministry of Manpower, the Ministry of Transport and other departments [7]. Japan established the Novel Coronavirus Pneumonia Countermeasures Headquarters, which led the command and control of COVID-19. The Cabinet Crisis Management Center and the Ministry of Health, Labour and Welfare as the main responsible body, and gradually strengthened the prevention and control mechanism [8].

At present, COVID-19 management is normalized, but the research on China's prevention and control of COVID-19 is relatively rare. In response to the status of the COVID-19 pandemic, the experience of prevention and control of COVID-19 pandemic in China and abroad is analyzed. The enlightenment from crisis prevention and control management is discussed, from aspects of public health literacy, monitoring and warning mechanism, disease prevention and control system, command and support system, and international cooperation. Effective measures are taken to prevent, respond quickly and control the crisis quickly. So as to provide guidance for the prevention and control of epidemic crisis in the future.

\section{The COVID-19 Global Pandemic}

In January 2020, novel coronavirus pneumonia was included in the management of class B infectious diseases and preventive measures for class $\mathrm{A}$ infectious diseases in China. The COVID-19 global pandemic was officially declared by the World Health Organization (WHO) in March 11th. At the time of writing, 34 provincial administrative regions in China and 219 countries and regions in the world have numbers of infected people. The global challenge of the COVID-19 pandemic crisis is becoming increasingly serious. As of August 2, 2021, 198,912,691 cumulative confirmed cases, 179,520,792 cumulative cured cases and 4,234,759 cumulative deaths have been reported worldwide, with a mortality / case ratio of $2.13 \%$ and a cure rate of $90.25 \%$ [9]. With the spread of novel coronavirus pneumonia, the number of confirmed cases worldwide has risen. At present, the number of deaths in Europe and North America is higher than that in Asia. The number of confirmed cases in the United States, India, Brazil, France and Russia ranks the five in the world.

The COVID-19 global pandemic crisis has the characteristics of a relatively long incubation period, wide coverage, fast transmission and difficult prevention and control [10]. Compared with other public crises, there are many uncertain influencing factors in the development process and evolution of the COVID-19 pandemic, which are difficult to predict, so it is difficult to prevent and control. COVID-19 prevention and control tend to be normalized. Meanwhile, it faces many challenges. As for the unknown origin of new coronavirus, some studies have suggested that the natural hosts of new coronavirus may be bats and Pangolins. The middle hosts may be mink, snakes, sea turtles, etc., and there is no clear conclusion [11]. New coronavirus spreads fast, its transmission places are complex. The main sources of infection are new coronavirus infected persons and asymptomatic new coronavirus infected persons. The main routes of transmission are respiratory tract droplet transmission, close contact transmission and aerosol transmission [12]. With the spread of new coronavirus, there have been many variants, which not only increase the risk of transmission, but also affect the accuracy of diagnostic testing. The spread of China's COVID-19 is basically blocked, and the risk of outbreak still exists due to the migration and aggregation of personnel. Local sporadic and small-scale aggregation of the epidemic often occurs. The emergence of asymptomatic infection and secondary infection makes the transmission more hidden. And the discovery also has limitations. The situation of COVID-19 in foreign countries is still grim. India, Indonesia, Brazil, Spain, the United Kingdom, the United States and other countries have increased the number of confirmed diagnoses to tens of thousands of people. It is a very important task to prevent and control imported foreign cases.

\section{Foreign Experience in COVID-19 Crisis Prevention and Control Management}

The COVID-19 pandemic affects many countries in the world, and no country is immune. The challenge is to ensure that all people are aware of and engage in the management of the pandemic. At present, although most countries have taken comprehensive measures according to the recommendations of WHO, the analysis of early response measures plays an important role in improving the national public health emergency mechanism and the emergency management ability of rapid handling of public health emergencies. Foreign experience in COVID-19 crisis prevention and control management is generally summarized as three main prevention and control strategies, namely, isolation, mitigation, and inhibition. 


\subsection{Isolation}

Isolation aims at blocking the spread of the virus, inhibiting the transmission chain and limiting the spread of the epidemic. China, Singapore, South Korea and Iran are all examples, with China as the typical representative. "Early detection, early report, early isolation and early treatment" was an important method for epidemic prevention and control in China [13]. China's key measure to block the spread of the virus was to resolutely and decisively close and block the channel from Wuhan. Wuhan was closed for 76 days. During the period, the authorities strictly managed cases and close contacts, increased social distance, suspended work, school, production, business, and prevented crowd gathering activities. The lessons learned included taking regional isolated measures, adhere to the prevention and control strategy of "external import and internal non-proliferation", cut off the source of infection as far as possible, and control the scope of the epidemic as far as possible [14]. After the outbreak of COVID-19, compared with other countries, South Korea's emergency response system started rapidly. Especially in epidemic detection, tracking, prevention and control and emergency disposal. South Korea adopted the prevention and control idea of "coping with excess is stronger than coping with delay". It blocked the inflow of novel coronavirus through special entry procedures, set up "Drive Thru", "Open Walk Thru", etc., carried out efficient and large-scale nucleic acid testing, implemented tracking management on confirmed cases, suspected cases and close contacts, and taken methods such as action restriction, hospital isolation or home isolation [6]. Singapore adopted strict early prevention and control measures against COVID-19, and imposed mandatory isolation for all immigrants for 14 days. The Ministry of Health set up a professional team to closely track patients' trajectories and their close contacts, and published the basic identity information and action track of confirmed cases in real time, so as to prevent further spread of the epidemic [15].

\subsection{Mitigation}

The goal of mitigation is not to put out novel coronavirus pneumonia and cut off the transmission, but to reduce Rt (Effective reproduction number) [16]. Germany, Italy and the United States are the main examples. Italy adopted delayed prevention and control measures [17]. First, it focused attention on the treatment of severe cases. Mild cases were isolated at home and observed, but it lacked necessary home supervision and management measures, which could not effectively block the spread of the virus. Second, increased social distance, prohibiting crowd gathering and protecting vulnerable groups. Third, due to the lack of protective materials, the Italian authorities only recommend three groups of people, including those with symptoms, to wear masks. Fourth, strengthening medical support, establishing temporary outdoor hospitals, and expropriating hotels and other places for isolation of patients. Compared with Italy, where the early epidemic situation was severe, Germany's early prevention and control response was simple. Germany adopted mitigation control measures, not limiting daily activities such as going out to work, hospital testing and shopping. Banning parties, encouraging home, cancelling or postponing large-scale activities, a key measure to mitigation strategy was to suggest that people with latent symptoms need be segregated at home and then go to the hospital if necessary [18]. The United States relied on people's self-monitoring and conscious home isolation. Mild patients were encouraged to heal themselves at home, and severe patients were treated intensively. Whether nucleic acid detection depended on the doctor's diagnosis, and the detection standard strictly limited [19].

\subsection{Inhibition}

Inhibition is to reduce the epidemic intensity to make the effective regeneration number $\mathrm{Rt}<1$. It is mainly carried out by isolating cases and close contacts, increasing interpersonal distance and stopping crowd gathering activities. "The hammer and the dance" strategy adopted by the Netherlands is a prime example [20]. The first stage is to take very strict measures in a relatively short period of time, such as limiting all aspects of public life, controlling the spread of the virus as soon as possible, to make the Rt below 1 . The second stage is a relatively stable period of lifting as many restrictions as possible while still maintaining low Rt, isolating and quarantining infected and high-risk groups, paying attention to social distance and disinfection and cleaning of public places, and prohibiting large-scale assembly [21].

\section{China's Experience in COVID-19 Pandemic Crisis Prevention and Control Management}

\subsection{The Whole Nation System}

From the patriotic health movement to SARS in 2003 and H1N1 in 2009, the national system has been a prominent feature of China's response to the public health crisis. In the prevention and control of the COVID-19, the whole nation system has also become a major advantage of China in distinguishing between Europe and the Americas. "Epidemic prevention and control need that the people of the whole country can work as one." The centralized and unified leadership of the Central Committee of the Communist Party of China has become the magic weapon of China to overcome the epidemic. In the early days of COVID-19, Hubei became the worst hit area. "When disaster struck, help came from all sides." The Central Committee of the Communist Party of China unified the allocation of human, material and financial support throughout the country. With the mobilization and participation of the whole society, the situation of epidemic prevention and control in Hubei province was quickly controlled. 


\subsection{Simultaneous Medical and Non-medical Measures}

Since outbreak of novel coronavirus pneumonia, the Chinese government has taken strict measures to prevent and control the disease. Based on modern medical technology, taking public health prevention and control as the main body. Meanwhile, medical and non-medical measures were taken, and non-medical public health prevention and control were paid attention. These measures effectively prevented the spread of the epidemic. In the medical part, on the basis of realizing the basic treatment in the hospital, the designated hospital, mobile cabin hospital and centralized isolation point were added for isolation treatment to solve the problem of admission and treatment. According to the "four early" principle, the policy of ensuring that all those in need were tested, isolated, hospitalized or treated was implemented. Traditional Chinese medicine treatment was willing to take as much as possible [22]. Through the integration of traditional Chinese medicine and western medicine, combined with modern medical technology, "one-to-one" precise treatment of critically ill patients, personalized treatment plan, and free treatment strategy were implemented. The novel coronavirus pneumonia prevention and control not only emphasizes medical treatment, but also was important for non-medical public health prevention and control. China has not only adopted traditional infectious disease prevention and control measures such as nucleic acid detection, personal safety protection, isolation and tracking, but also implemented new measures such as increasing social distance and travel restrictions according to China's national conditions. In order to curb the spread of COVID-19, Japan, South Korea, France, Italy and the United Kingdom have learned from China's experience and adopted various non-medical epidemic prevention and control measures.

\subsection{Timely, Transparent and Open Information}

Due to the lack of relevant experience in dealing with the public health crisis, the government did not disclose accurate epidemic information in time and guide the masses correctly in the early stage of SARS outbreak, which led to the panic and worry of the masses and the "salt snatching incident"[23]. Drawing lessons from SARS, in the COVID-19, in line with the principle of "early, fast and transparent", experts and officials directly faced the media and the public, actively informed WHO and the international community of the epidemic situation, timely issued a series of effective prevention and control measures, and shared scientific data and prevention and control experience. This reduced social panic and improved social trust.

\subsection{Efficient Joint Prevention and Control Mechanism}

Joint Prevention and Control Mechanism of the State Council under the direct leadership of the Central Committee of the Communist Party of China and the State Council was adopted to deal with COVID-19. It was composed of 32 departments, including epidemic prevention and control, medical treatment, scientific research, publicity, foreign affairs, logistics support, and forward work. The State Council Joint Prevention and Control System integrated team timely reported the COVID-19 situation to the provincial (region, municipal) joint prevention and control mechanism, leading group, headquarters. Disease control, police, communications, transportation, border inspection, customs, aviation, railway, health and other departments coordinated to ensure fit operation [24].

\subsection{Cadre Accountability System}

The cadre accountability system is the characteristic system of China's COVID-19 crisis prevention and control management. In response to the severe situation of the epidemic, the Central Committee of the Communist Party of China severely investigated and punished such disciplinary problems as failure to take responsibility, failure to act, negative response, and dereliction of duty in epidemic prevention and control work. In accordance with the law, discipline inspection and supervision authorities at all levels resolutely punished officials who were derelict in their duties, and carried out precise supervision over the enforcement of discipline and law, ensuring that normal epidemic prevention and control work was carried out with high quality.

\section{Enlightenment of COVID-19 Pandemic Crisis Prevention and Control Management}

Fundamentally, of the major prevention and control measures taken by countries around the world to cope with the COVID-19 pandemic crisis, isolation is the ultimate means of prevention and control. The COVID-19 pandemic has had a profound impact on China's society in an encompassing way. It puts forward new requirements for government departments, grass-roots medical and health institutions, scientific research institutions, news media and the public. At present, the spread of the novel coronavirus pneumonia in many countries is accelerating, so the risk of importing into China is gradually increasing. Crisis prevention and control management of the COVID-19 pandemic in China develops systematically, to scientifically take effective measures to prevent, respond and control the crisis.

\subsection{Mobilize the Whole People to Improve Public Health Literacy}

Whether it is nationwide mobilization under the leadership of the Chinese government, or the cooperation between the governments of other countries and non-governmental organizations, in the face of large-scale public health epidemics, all of them require comprehensive mobilization, active defense, and strengthening of public health literacy. The improvement of public health literacy is inseparable from the support and guidance of national government agencies. When new infectious diseases occur, the public is 
not fully aware of the extent of its harm. In addition, the government's propaganda, publicity and education on the characteristics of public health crisis, transmission channels, and harmful consequences are not enough, resulting in the public not participating in the crisis management process of public health events well. The COVID-19 pandemic poses a huge threat to the public's physical and mental health. It not only causes panic and fear [25], but also presents serious problems such as strong psychological stress reaction and multiple psychological problems. In response to this situation, on the one hand, taking the masses as the foundation, grasping the propaganda points, and intensifying the knowledge propaganda and education work on danger, severity and importance of public health crisis [26]. Through the government website column, WeChat official account, online epidemic prevention lecture, and epidemic prevention manual, to popularize the measures and information of joint prevention and control of all regions and departments. Prevention and control measures are timely, accurate and high-density transmitted to the public to establish public crisis awareness [27]; On the other hand, through building online multi-party communication platform and forming multi-level information and resources sharing, so as to realize the joint participation of the government, the public, hospitals, news media and other forces in the management of prevention and control, and give full play to the important role of the public as the main body of COVID-19's prevention and control.

\subsection{Establishing Public Health Crisis Prevention and Control Platform and Improving Monitoring and Early Warning Mechanism}

Sensitive monitoring system is the premise of timely detection of disease [28]. The incubation period is different with different microorganisms. The novel coronavirus pneumonia infectors may not display obvious symptoms during the incubation period. Based on current epidemiological investigation, the incubation period is of 1-14 days, mostly 3-7 days [29]. It has brought great difficulty to medical institutions in timely detection and coping with the novel coronavirus pneumonia. Improving the monitoring and early warning mechanism of unknown infectious diseases is an effective way to prevent the spread and deterioration of a public health crisis. The monitoring and early warning mechanism of a public health crisis includes three modules: crisis identification, crisis assessment, and crisis prevention and control, which are specifically divided into early warning index system, early warning information system, emergency response system, resource sharing system, information notification mechanism, etc. The possible public health crisis is classified and graded in advance, and the risk is quantitatively analyzed and evaluated. When the crisis occurs, judging the early warning, monitoring the whole process, formulating a detailed prevention and control plan in time, taking quick response measures, and conveying the relevant information to all departments quickly, accurately and efficiently, so as to improve the ability of emergency linkage.

\subsection{Establishing a Strict Disease Prevention and Control System and Implementing Prevention and Control Measures}

The key construction of the disease prevention and control system is not only the implementation of prevention and control measures, but also the improvement of the ability of the basic public health prevention and control forces and public health prevention and control institutions.

The implementation of public health crisis prevention and control countermeasures mainly includes the following three risk points. First, prevent overseas importation strictly. The customs strengthen sampling inspection, and quarantine of imported goods such as frozen food, especially the outer packaging and the goods themselves. The epidemic prevention departments also strengthen detection, preventive cleaning and disinfection, and improve the inspection system of cold chain food and other goods. Second, improve the safety protection level of high-risk groups [30]. All employees should be strictly managed, carried out health monitoring, etc. Personal occupational protection awareness is supposed to be strengthened to ensure that employees are well protected. Third, formulate daily prevention and control measures for high-risk places. Implement disease prevention and control measures in collective places, such as hospitals, community centers, clinics, designated medical institutions and schools. The places are regularly cleaned and disinfected, and improved the sanitary conditions. In the COVID-19 pandemic, medical treatment is the most dangerous and vulnerable place for novel coronavirus pneumonia. The prevention and control of sites, personnel and facilities is the key. The storage, processing, transportation and sales of frozen products are also high-risk places for virus transmission and amplification. The inspection and monitoring of markets and enterprises such as seafood market, frozen seafood processing enterprises need to be strengthened.

The improvement of professional technology of grass-roots public health prevention and control forces is the necessary prerequisite and guarantee for the implementation of scientific management and control of risk areas. Setting up professional security teams of public health in streets, communities and villages, carrying out emergency drills regularly, and training public health crisis prevention and control skills to ensure the correct implementation of prevention and control policies. National centers for disease control and prevention at all levels set up expert groups for public health crisis prevention and control to cultivate a group of high-level, compound and applied command and technical personnel according to the actual needs, and strengthen the allocation of relevant infrastructure.

\subsection{Establishing a Powerful Command and Support System, Jointing Multi-department Cooperation and Strengthening International Cooperation}

In order to effectively reduce the harm of public health 
crisis and realize effective management and control of possible public health crisis, a government-led, cross-departmental and regional command and coordination system should be established in a holistic way [31]. And a public health crisis prevention and control platform need to be established with the cooperation of emergency departments, medical and health departments, scientific research institutions and other units. A leading group for emergency response to public health crisis need to be set up to coordinate the issues such as shortage of emergency supplies. At the same time, the prevention and control platform integrates big data of public health events, then timely and dynamically releases authoritative information such as command and dispatch information, prevention and control measures, and government policies. In view of the possible emerging infectious disease crisis, the society formulates a reasonable and effective emergency plan in advance and establishes a rapid expansion mechanism, providing support services for scientific prevention and control.

In the face of the COVID-19 pandemic, the government constantly improves the detection ability of medical institutions, and makes good normalization and precise prevention and control. It also sets up isolation points to isolate treatment cases, and management of close contacts, to improve the screening findings more strictly, more closely and finely. Scientific research institutions increase medical research and development, and actively carry out vaccination research. In order to meet the actual needs of the epidemic situation, the emergency medical assistance system is improved from the aspects of cross regional medical treatment, the diversity of drug types, and the convenience of settlement methods. Medical security should be comprehensive and fair. Based on the post responsibilities and professional characteristics of the team, the emergency rescue team, as a backup support team, makes overall plans for epidemic prevention and control and emergency rescue work, and is always ready to rapidly launch the "Resistance War" of epidemic prevention and control [32].

Another experience is to strengthen international cooperation and share anti epidemic experience. At the beginning of the COVID-19, China was helped and supported by many countries and peoples around the world, fighting against the epidemic with international cooperation. Then China took the responsibility of the great powers, and shared information in a timely, open and transparent manner, contributing to the international community's measures and experience in preventing and controlling the epidemic. So far, China has provided medical material assistance or fund donation to more than 160 countries, regions and international organizations, such as masks, protective clothing and testing reagents. China also provided vaccine assistance and support to more than 100 countries, regions and international organizations [33]. Multi-faceted and multi-level cooperation among countries around the world is the key to fight the epidemic. It is of great practical significance to build a community of common destiny for mankind to overcome the epidemic situation together.

\section{Conclusion}

The COVID-19 pandemic has caused great threat to the safety and health of the world's people. To reduce the risk of the novel coronavirus pneumonia, the development of science and technology must be paid attention. It is of great importance to uphold the idea of the community of human destiny and accelerate the development of vaccines and clinical trials of drugs. The COVID-19 pandemic spread widely and rapidly. In order to prevent and control the epidemic, many countries have taken isolation, mitigation, and inhibition measures according to the actual situation. For China, the national system, joint prevention and control mechanism, cadre accountability system, simultaneous medical and non-medical measures, timely and transparent information disclosure and other prevention and control experience have played a key role. It is of great significance for some countries with weak health system to learn from China's experience coping with the epidemic and effectively curb the spread of the epidemic. And some enlightenments are discussed to better improve the management system and ability of public health crisis prevention and control lays a foundation. Firstly, to mobilize the whole people to improve public health literacy. Secondly, to establish public health crisis prevention and control platform and improve monitoring and early warning mechanism. Thirdly, to establish a strict disease prevention and control system and implement prevention and control measures. Fourthly, to establish a strong command and support system, joint multi-department cooperation and strengthen international cooperation.

\section{Conflicts of Interest}

The authors declare they have no conflicts of interest.

\section{References}

[1] Gao GF. From "A" IV to "Z" IKV: attacks from emerging and re-emerging pathogens. Cell, 2018, Volume 172, 1157-1159.

[2] N. P. A. S. Johnson, J. Mueller. Updating the accounts: global mortality of the 1918-1920 "Spanish" influenza pandemic. Bull History Med, 1918, Volume 76, 105-115.

[3] C. Scholtissek, W. Rohde, VonV. Hoyningen, R. Rott. On the origin of the human influenza virus subtypes $\mathrm{H} 2 \mathrm{~N} 2$ and $\mathrm{H} 3 \mathrm{~N} 2$. Virology, 1978, Volume 87 (1), 13-20.

[4] L. A. Reperant, A. D. M. E. Osterhaus. AIDS, Avian flu, SARS, MERS, Ebola, Zika... what next? Vaccine, 2017, Volume 35 (35), 4470-4474.

[5] Qiu AJ, Cui X. Novel coronavirus pneumonia emergency mechanism and its inspiration in South Korea: Taking the practice of coping with the new crown pneumonia as an example [J]. Cities and disaster reduction, 2021, Volume $\{4\}$ (01): 1-4. 
[6] Ye J. South Korea's novel coronavirus pneumonia measures: analysis and inspiration [J]. Outlook on global science, technology and economy, 2020, Volume 35 (07): 60-68.

[7] Ren H. Singapore's novel coronavirus pneumonia epidemic prevention and control mode and its evolution [J]. Research on Yunda area, 2020, Volume $\{4\}$ (02): 109-129+225.

[8] Cui YY, Li H. Japan's emergency management mechanism seen from COVID-19 [J]. Research on Japan, 2020, Volume 34 (06): 8-14

[9] Tracking COVID-19's global spread [DB/OL]. [2021-5-6]. https://edition.cnn.com/interactive/2020/health/coronavirus-m aps-and-cases/.

[10] Liu SL. Novel coronavirus pneumonia epidemic situation and coping strategies. China Development Observation, 2020, Volume Z3, 13-17.

[11] Huang YN, YN, Wang L, Cao MX, Huang ZC, etc. Research Progress on environmental transmission of sars-cov-2 [J/OL]. Environmental Chemistry, Volume 1-13 [2021-08-02]. http://kns.cnki.net/kcms/detail/11.1844.x.20210708.1626.040. html.

[12] General Office of National Health Commission. New Coronavirus pneumonia diagnosis and treatment plan (trial version fifth) [ER/OL] (2020-02-05) [2021-7-15]. http://www.gov.cn/zhengce/zhengceku/2020-08/19/content_55 35757.htm.

[13] Notification of the State Council on the Joint Prevention and Control Mechanism for Novel Coronavirus Infection Pneumonia on Further Improving the Prevention and Control of the Current COVID-19 Epidemic [J]. Gazette of the State Council of the People's Republic of China, 2021, Volume (04): 13-15.

[14] Lai SJ, N. W. Ruktanonchai, Zhou LC, O. Prosper, Luo W, J. R. Floyd, et al. Effect of non-pharmaceutical interventions to contain COVID-19 in China. Nature: International weekly journal of science, 2020, Volume 585 (7825), 410-413.

[15] Liu JJ, Zhang SQ, Song MK. Novel coronavirus pneumonia and coping strategies in 7 countries such as China in early 2020 [J/OL]. Shanghai preventive medicine, 1-11, [2021-05-08]. http://kns.cnki.net/kcms/detail/31.1635.R.20210208.1406.003. html.

[16] S. Eubank, I. Eckstrand, B. Lewis, S. Venkatramanan, M. Marathe, C. L. Barrett. Commentary on Ferguson, et al., "Impact of Non-pharmaceutical Interventions (NPIs) to Reduce COVID-19 Mortality and Healthcare Demand". Bulletin of Mathematical Biology: A journal devoted to research at the interface of the life and mathematical sciences, 2020, Volume 82 (4), 52.

[17] C. Vicentini, V. Bordino, P. Gardois, C. M. Zotti. Early assessment of the impact of mitigation measures on the COVID-19 outbreak in Italy. Public health, 2020, Volume 185, 99-101.

[18] Li C. COVID-19's prevention and control measures in Germany [J]. International research reference, 2020, Volume (05): 9-14.
[19] Ping W. Coping with the novel coronavirus pneumonia crisis in the US [J]. Peace and development, 2020, Volume (03): $18-37+132-133$.

[20] Panagopoulos Andreas, Altmeyer Matthias. The Hammer and the Dance of Cell Cycle Control [J]. Trends in Biochemical Sciences, 2020 (prepublish).

[21] G. Antonides, E. V. Leeuwen. COVID-19 crisis in the Netherlands: "Only together we can control Corona" [Internet], 2020, DOI: https://doi.org/10.1007/s11299-020-00257-x. Available from: Mind \& Society.

[22] Information Office of the State Council of the people's Republic of China. China's action against the novel coronavirus pneumonia epidemic [R/OL]. (2020-6-7) [2021-5-6].

http://www.gov.cn/zhengce/2020-06/07/content_5517737.htm.

[23] C. Allen. Back to the future: lessons of a SARS hysteria for the COVID-19 pandemic [J]. Cultural Studies, 2021, Volume $35(2-3)$.

[24] Guiding Opinions of the State Council for the Joint Prevention and Control Mechanism of Novel Coronavirus Infected Pneumonia on Normalized Prevention and Control of COVID-19 Epidemic [J]. Gazette of the State Council of the People's Republic of China, 2020, Volume (14): 15-17.

[25] Cheng L, Zheng LP, Yan S, Fan X. Anxiety status and related factors in patients with coronavirus disease 2019. Zhejiang Medical Journal, 2020, Volume 42 (04), 315-317.

[26] M. Y. Ali, R. Bhatti. COVID-19 (Coronavirus) Pandemic: Information Sources Channels for the Public Health Awareness. Asia Pacific Journal of Public Health, 2020, Volume 32 (4), 168-169.

[27] Luo L, Zeng XJ, Liao X, Yang YQ. Disease cognition, coping style and exercise behavior among the public during novel coronavirus epidemic: an online survey. Chinese Journal of Public Health, 2020, Volume 36 (02), 156-159.

[28] Dai YY, Wang JM. Identifying the outbreak signal of COVID-19 before the response of the traditional disease monitoring system. PLoS neglected tropical diseases, 2020, Volume 14 (10), e0008758.

[29] You YL, Fang DX, Xu M. Nursing care of 1 novel coronavirus infection patients with pulmonary mass occupying latent period. Chinese General Practice Nursing, 2020, Volume 18 (5), 563-565.

[30] C. Gordon, A. Thompson. Use of personal protective equipment during the COVID-19 pandemic. British Journal of Nursing, 2020, Volume 29 (13), 748-752.

[31] J. Cook. Incident Command in the Time of COVID-19. Laboratory medicine, 2020, Volume 51 (6), e78-e82.

[32] Wang W, Zhang LA, Hou YX, Fu RY. Emergency Response Capability and Operation Management of Fire and Rescue Forces during COVID-19. Journal of the Armed Police Academy, 2020, Volume 36 (06), 62-65.

[33] Bao JY. China's strength and speed in global epidemic prevention and control [J]. People's Forum, 2020, Volume (Z2): 40-43. 